\title{
Fermilab
}

TM-1406

1501.000

OFFSET COLL DESIGNS FOR SUPERCONDUCTING MAGNETS, A LOGICAL DEVELOPMENT

T. Collins

March 1986 


\section{OFFSET COIL DESIGNS \\ for SUPERCONDUCTING MAGNETS, \\ a Logical Development.}

T. L. Collins

Fermilab, March 1986.

Dipoles and quadrupoles for any new, large proton ring must be stronger, smaller and have better field shape (systematic error) than those used in the Doubler. The present two-shell designs are rigid in that the coils are too thin but cannot be relatively fatter without destroying the field quality.

An examination of the coil shapes for dipoles and quadrupoles which produce perfect fields from a uniform current density shows clearly that our presistent use of a circular form for the inner surface of the coils is a poor approximation. When this is corrected by "offsets" there is a striking improvement both in the strength of fields and in the field quality.

The same analysis makes clear that the efficient use of superconductor and the overall magnet size is determined by the perfect coil shapes. Any reasonable magnet will not differ significantly from the ideal for these parameters. This will be particularly helpful in setting design goals for very large quadrupoles.

The offset two-shell dipole design preserves the mechanical features of the highly sucessful, resilient doubler magnets while greatly extending the performance. For a quick preview you should compare figures 3 and 5 and the adjacent performance characteristics (definitions on pg. 3 ). 


\section{DIPOLES}

Smaller superconduting dipole magnets for large proton rings must have both relatively larger coils and relatively much higher design-field quality. This paper examines this design problem in a logical manner, producing a new form of two-shell design which is a large step in the required direction, howover the most important contribution is that one can clearly see the limits for practical coil design. Better designs are possible - ones that have desirable construction features and maintain or improve the field quality - but they will not differ substantially in efficient use of superconductor or overall size.

We will be concerned with the effect of changes in coil shape, independent of the actual size. For this purpose we use a scale, a, which is the actual horizontal distance to the inner surface of the coil (the inner radius for familiar designs) and measure everything relative to this unit. Typically $\mathbf{a}=2-4 \mathrm{~cm}$.

Practical dipole magnets are never perfect. There are random errors, random from magnet to magnet, from construction variance. There is also a systematic deviation of the field which is a property of the designed coil shape. Systematic field deviation is usually expressed in terms of multipole coefficients (relative to Bo) which, by symmetry, are limited to the normal multipole sequence: 6-, 10-, 14-, 18-, 22-pole, etc. The lowest three of these multipoles can be related to specific shape factors in the coil design and are easily set to zero. The higher multipoles are a "package" that represent, in a complicated way, the strong but smooth deviation of the field close to the coil.

We will use relative multipole coefficients which are expressed in units of a. This produces numbers of a convenient size, for example the relative bre, bas, and b2s for doubler magnets are $-.033,+.029$, and -.013 (a typical pattern).

It is important to remember that the upper limit to the higher multipoles from beam dynamics is a set of absolute values, and for the same beam behaviour the relative values will increase with $\mathbf{a}^{8}$ (18-pole). Because of the high powers of $a$, changes in the multipoles must be large to be significant. 
Setting a reasonable limit on systematic multipoles is a complex business, and is not the subject of this paper, but some comments are in order. Systematic field shape does limit the performance of the Doubler and was the primary consideration in the choice of the coil design. For any ring that is larger, or more complex (less symmetric) one can anticipate that the necessary systematic field quality will determine the size and shape of the coils.

Too often it has been assumed that the "good"" beam size simply scales with the dipole size (a). Theoretically this cannot be true. There is no rigorous scaling rule and one must resort to extensive tracking studies which must include correction sextupole patterns and random field errors because they interact strongly. Some studies show that beam scaling is close to $\mathbf{a}^{2}$.

Studies by the author on a simplified version of the SSC indicate that an upper limit to the relative values for the higher multipoles is .003 with $a=2.5 \mathrm{~cm}$. (a $5 \mathrm{~cm}$ dipole). This value will be used as a realistic example but it is not intended as a definitive limit.

In this analysis close iron is ignored. If the iron is restricted to its linear range (B<20kG) then it will contribute only a small increase in $B_{0}-$ perhaps $20 \%$ - which will accordingly reduce higher multipole coefficients by a not particularly significant amount. If the iron is highly saturated then an adequate field quality at all fields cannot be presumed.

Some useful scaled parameters describing the performance of a shape are:

$$
\begin{array}{ll}
\text { b a "field", where } & B_{0}=b\left[\mu_{0} j a / 2 \pi\right] \text { tesla, } \\
q \text { a "cross-section" } & \mathbf{q a}^{2} \text { is the coil area }\left(\mathrm{cm}^{2}\right), \\
\mathbf{f} \text { an "efficiency" factor } & \mathbf{f}=\mathbf{b} / \mathbf{q}, \\
\mathbf{R} \text { a "size" } & \mathbf{R a}=\text { radius of a circle circumscribing the coil. }
\end{array}
$$

The current density $j$ is an average over the whole coil area, which includes insulation and other "waste" space as well as copper. It is considerably reduced from $j_{0}$, the $\mathrm{Nb}-\mathrm{Ti}$ filament current density [typically $j_{0}=2400$ and $\left.\mathrm{j}=500-800 \mathrm{a} / \mathrm{mm}^{2}\right]$.

We can now restate the opening sentence. It is very desirable to reduce the overall size (Ra) and cost of dipoles. A reduction in the internal size (a) however requires an increased relative field (b) and a fatter coil (a), while improving the relative values of the higher multipoles in order to maintain an acceptable absolute field quality $\left(a^{-0}\right)$. 


\section{A Logic for High-Quality Coil Design}

The field quality we need is very high, typically a deviation of 1 in $10^{5}$ is significant. It is useful to start with the coil shape which gives a perfect field for a uniform current density; this is shown in figure 1.

There is an unfortunate tendency to include this shape (and all shell designs) in the name cosine-theta. Properly that term applies only to a ring coil with a current density that varies as cosine theta, which is painful to construct from turns to the accuracy needed. The coil in figure 1 does have a width that varies as cosine theta but that is not nearly a sufficient criterion for a high quality field. What is unfortunate is that the improper name has diverted attention from the proper, very specific shape.

The perfect coil shape is the crescents created by a pair of overlapping circles. The shape is specified by the relative offset of the circles from the center (d).

The parameters for this coil are

$$
\begin{aligned}
b & =2 \pi d \\
q=4\left[d(1+2 d)^{2 / 2}\right. & \left.+(1+d)^{2} \sin ^{-1}(d / 1+d)\right] \\
R & =1+2 d \\
f & =b / q \sim .6
\end{aligned}
$$

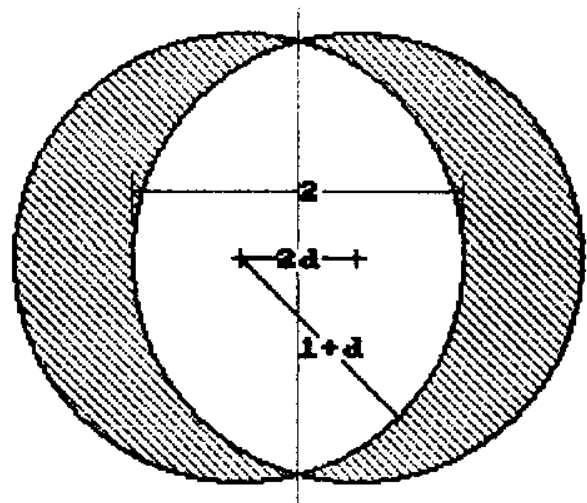

figure 1 The Perfect Coil

Table I lists parameters for this design for comparison with actual coils. We will find a suprisingly close agreement, particularly with coil degigns of higher quality.

It is often assumed that if one designs a coil in a sufficient number of independent "chunks" then one can vary the dimensions until many multipoles are reduced to zero. This process inevitably degenerates after a few (or less) multipoles, to one of trying to squeeze a balloon. The reason is simple.

The overlapped circles are the only perfect solution. For the high relative quality required for large rings with small dipoles we must use designs which closely approximate this shape. In particular missing bita from the ideal shape must be compensated nearby - gaps along the inner surface (dummy turns) cannot be expected to work well. We can be sure that the efficiency $(f)$ and the overall size $(R)$ will be close to table I values. 


\section{Present Designs}

The gingle shell coil shown in figure 2 is the progenitor of the doubler design. This is a last-century design in which the sextupole is set to zero. It has good field quality, not sufficient for ring dipoles but excellent for other purposes such as steering dipoles.

Superimposed on the coil is the outline of the perfect coil with the same inner dimension (a) and the same relative field (b). This figure is drawn for the same $b$ as the next figure, which is the standard two-shell.

The angles for the two shells are chosen to make 6- and 10-pole zero, which is possible if the shell thickness $(w)$ is less than .3.

If $w=.1735$ then the 14 -pole is also zero, and this is size in the figure. Then

$$
b=1.105, \quad f=.704,
$$

rel. multipoles $-.0338,+.0337,-.0161$

The equivalent perfect coil has $f=.670$.

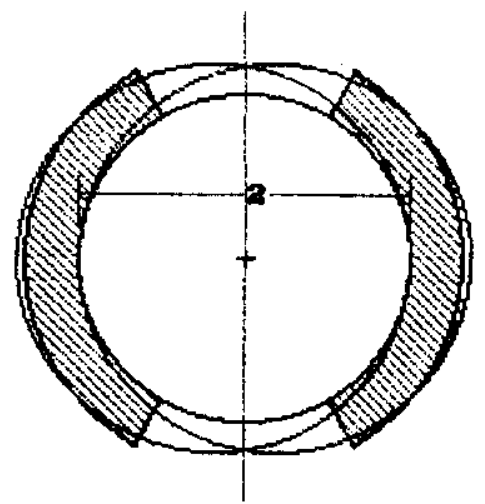

figure 2 2/3 Rule Design

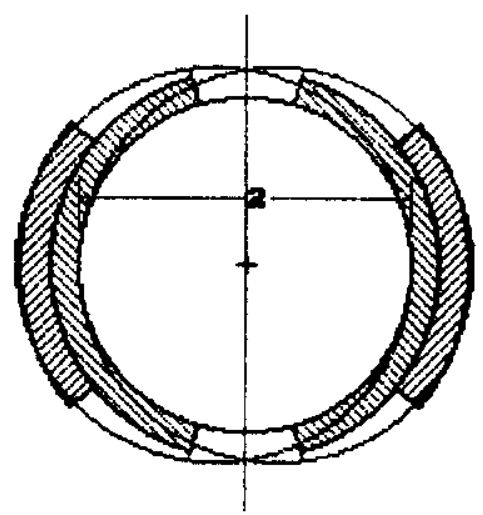

figure 3 Two-Shell Design

The Doubler used $w \sim .2$, but the resultant 14-pole would be objectionable in smaller dipoles or larger rings. Essentially the shape of this design is fixed, and the relative field (b) is too low and the relative multipoles too high for our purpose, although it is adequate for larger dipoles and smaller rings.

A brief examination of the fit to the perfect coil is sufficient to make the next design step embarassingly obvious - we should change the inside shape to conform more to the offset circles! The coils are not mounted on a pipe, they are supported externally by collars built from die stamped laminations so a different inside shape is quite practical. We will use shells which on each side are concentric (and use keystoned turns) but the two sides will be offeet so the centers overlap (by 2d). An appropriate generic name for this design is offet shells. 
offset Shells - The Three-Shell

We start with a design that we can anticipate will be excellent: three concentric shells on a side, each of thickness $w$, and an offset such that $2 d=3 w$ which will give an excellent fit for the inside surface.

For each w and $d$ one adjusts the three angles to make the lower multipoles zero. Now there is no problem with numerology and any wirth is possible. Figure 4 is drawn for

$$
w=.2 \text { then } b=1.95, f=.607
$$

$$
\text { multipoles }-.0021,+.0005,+.0001
$$

The equivalent perfect coil has $f=.605$.

The shells fit nicely within a circumscribed circle (R) which is the best measure of the overall size.

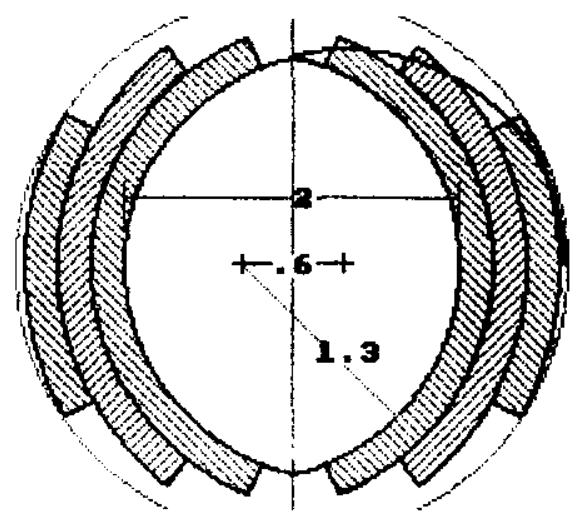

figure 4 The offset $3-$ Shell

This is indeed an exellent design, but for factory production on a large scale it is expensive. Table II gives dimensions and parmeters for a full range of $b$. It is not anticipated that dipoles will actually be built to these specifications, in general one will choose a simpler design using more material, but table II provides a reasonable upper limit of quality and complexity for comparison.

There is much more to producing a practical design than cross-hatching coil outlines. Turns should have a substantial thickness and insulation is not negligible, however shells using key-stoned turns do perform close to the simplified designs. This is not true of all construction styles.

A single stack of flat turns which are slid over against a circular side can, for very thin turns, accurately reproduce the perfect coil up to the crossover. The little caps beyond the crossovers are very important and must be approximated. A rectangular shape with $2 / 3$ the width of the main turns is a good approximation, but this is a real complication because the caps will require a different cable size and are therefore separate coils. The biggest problem starts when one considers a real turn thickness and the oblique angles between the turns and the circular side near the crossover. The approximation to a smooth inner surface becomes extreme and the field quality is much degraded. These paper difficulties become worse in actual hardware and the "simple" slipped stack is complicated and far from "perfect". 
Offset Shells - The Two-Shell Design

In these designs we assume an offset (d) and adjust the two coil angles plus the shell width $(w)$ to set the three lower multipoles to zero. The inner coil surface does not correspond to the equivalent perfect coil and we do not know a priori that there will be much improvement. However to appreciate the effect of a small offet compare figure 5 with figure 3 . This coil has

$$
b=2.165, f=.607
$$

multipoles $-.0076,+.0062,-.0027$.

Not the quality of the 3-shell but an enormous improvement over the previous designs. We can build high fields with improved quality while preserving the advantages of the twoshell construction. See table III for a set of designs.

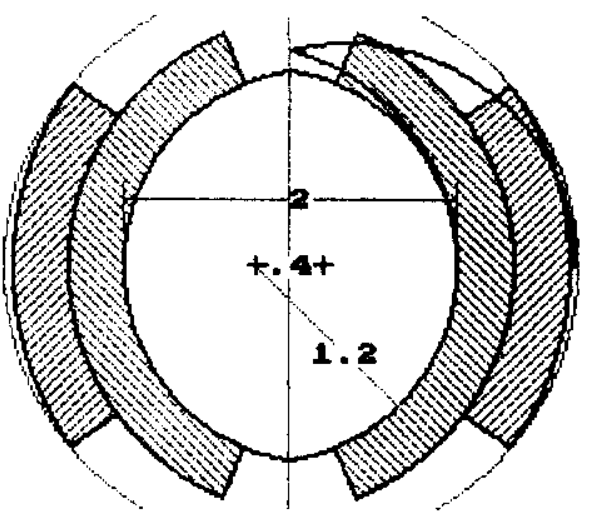

figure 5 An offset Two-Shell

\section{Variations on the Design}

This design uses a different cable for the outer shell. In this case the turns are 1.25 times thicker and 0.8 as wide and the shells have the same current density $(j)$, which is a good compromise between improved quality and efficiency. (Higher $j$ reduces the quality). The multipoles are

$$
-.0049,+.0033,-.0008
$$

which will turn out to be a somewhat marginal improvement, but may be of advantage for

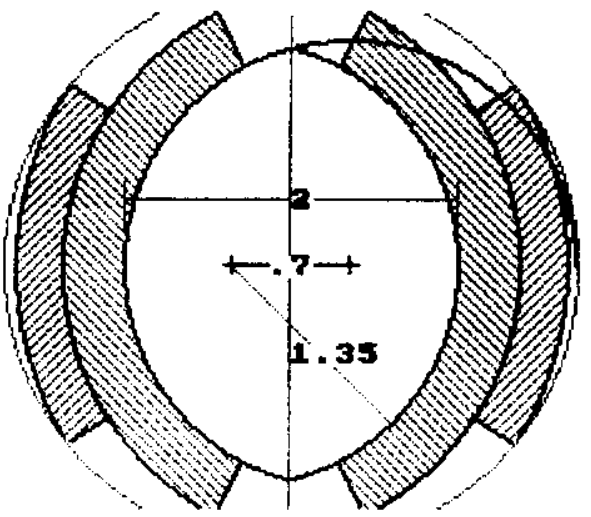

figure 6 Different Cables large scale production where two wire sizes involve little extra expense. There are many choices for the relative shell sizes and it is not perticularly important that the current densities be the same. Table IV lists designs with the outer width 0.7 times the inner and the same $j$.

An important consideration as coils become wider is the difficulty of producing cable with a wedge shape, called keystone. A measure of the keystone is the fractional reduction in the inner edge (or increase in the outer) compared to the average thickness. For doubler coils the keystone (including insulation) was about .1. The difficulty increases rapidly with this parameter so the reduction of keystone generated by the offset is most welcome. Tables III and IV have a column for "kstn". 
Good Things about Shell Designs

The doubler two-shell coils with their particular design of an external supporting collar have withstood the tunnel wars without significant voltage breakdown, neither turn-to-turn nor ground faults. This is as it should be, but in the long history of proton accelerators this is not the general case for new types of coils. Superconducting magnets prior to the doubler amasged a dismal breakdown record. There are design guidelines for avoiding these problems.

A voltage breakdown is literally a break caused by excessive local stress which comes from both mechanical and electrical forces. An insulator subjected to mechanical stress will break at a correspondingly lower voltage. Materials with high internal stresses such as sheet glass or epoxy are very unreliable insulators.

Avoiding high electrical stress concentrations is the easy part. Avoiding inadvertant mechanical stress concentrations has never been easy, but at least we can avoid obvious ones. In the doubler design there is a small amount of B-stage epoxy between the wrapped turns. In addition to bonding the coil for handling, this material provides a cushion to eliminate point contacts which would seriously degrade the turn-to-turn insulation.

The magnetic forces on push the coil outward against the firm continuous support of the collars, in addition they compress the shells azimuthally. The coils are built a little oversize so that the collars provide a pre-load to the ends of the shells which just overcomes the compressive forces and prevents coil motion. The coils must be sufficiently oversize and compliant so that slightly smaller coils have adequate pre-load and slightly larger coils are not overstressed. The direction of the pre-load, the wedge shape of the turns and the compliant material combine to firmly, but gently seat each turn against the outer support. One cannot count on the magnetic forces to seat the coil because cold insulation and bonding materials are rigid and brittle and local stress concentrations are inevitable.

One does not lightly abandon a highly successful coil type for a "new, improved" one. This is not to denigrate the capabilities of magnet designers, but none of us can fully predict the development of mechanical stress concentration after a year or two of operation. 


\section{Designing for Quality - A Different Proceedure}

Let us assume that we want a 6 tesla dipole with absolute quality corresponding to relative higher multipoles of .003 at $\mathbf{a}=2.5 \mathrm{~cm}$. First we use the three-shell table to find:

$b=1.8, a=2.5 \mathrm{~cm}, j=667 \mathrm{a} / \mathrm{mm}^{2}, q a^{2}=18 \mathrm{~cm}^{2}, N b-T i=5.1 \mathrm{~cm}^{2}, R a=4.0 \mathrm{~cm}$ The $\mathrm{Nb}-\mathrm{Ti}$ area as8umes $2400 \mathrm{a} / \mathrm{mm}^{2}$ in the filaments. (I assume that $667 \mathrm{a} / \mathrm{mm}^{2}$ is a reasonable overall current density for a 3 -shell). Suppose we have an additional restraint that a must be $2 \mathrm{~cm}$. For the same current density we would need a minimum $b=2.25$, but the relative multipoles now must be less than .0005 , which is beyond the quality level of even a threeshell for that b. We could use

$b=3, a=2 \mathrm{~cm}, j=500 \mathrm{a} / \mathrm{mm}^{2}, q a^{2}=22 \mathrm{~cm}^{2}, \mathrm{Nb}-\mathrm{Ti}=4.6 \mathrm{~cm}^{2}, \mathrm{Ra}=4.0 \mathrm{~cm}$,

where the lower current density is achieved by adding copper to the superconductor. This dipole has the same external size as the first one - which is what most of us mean by "size" - and if anything is a little more expensive. The first one undoubtedly has lower random field errors so the correct choice is obvious.

We turn now to the two-shell table which we convert to a table of dipoles with different internal size a but with the same absolute quality as above.

\begin{tabular}{|c|c|c|c|c|c|c|c|c|c|c|c|c|}
\hline b & $\begin{array}{l}i \\
i\end{array}$ & $\begin{array}{c}\mathbf{a} \\
\mathbf{c m} .\end{array}$ & $\begin{array}{l}\vdots \\
\vdots\end{array}$ & $\underset{\mathbf{a} / \mathrm{mm}^{2}}{\mathbf{j}}$ & : & $\begin{array}{l}q a^{2} \\
c m^{2}\end{array}$ & $\vdots$ & $\begin{array}{c}N b-T i \\
c a^{2}\end{array}$ & $\begin{array}{l}\vdots \\
\vdots\end{array}$ & $\begin{array}{l}\mathrm{Ra} \\
\mathrm{CE}\end{array}$ & i & $k \in \operatorname{tn}$ \\
\hline & & & & & & & 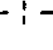 & & & & $i$ & n \\
\hline 1.2 & : & 3.32 & $i$ & 753 & $!$ & 19.0 & i & 6.0 & $i$ & 4.6 & i & .09 \\
\hline 1.4 & : & 3.20 & : & 670 & : & 21.3 & : & 5.9 & $\vdots$ & 4.6 & : & .09 \\
\hline 1.6 & : & 3.08 & i & 608 & : & 23.2 & : & 5.9 & $:$ & 4.7 & $i$ & .10 \\
\hline 1.8 & $i$ & 2.98 & : & 559 & : & 25.1 & : & 5.9 & : & 4.7 & i & .11 \\
\hline 2.0 & i & 2.88 & $!$ & 520 & ; & 26.9 & i & 5.8 & : & 4.8 & $i$ & .11 \\
\hline & $i$ & & $i$ & & : & & 1 & & $i$ & & ; & \\
\hline 2.2 & i & 2.79 & $:$ & 488 & $i$ & 28.4 & : & 5.8 & $:$ & 4.8 & : & .12 \\
\hline 2.4 & ! & 2.71 & $!$ & 461 & $i$ & 29.9 & ; & 5.8 & : & 4.9 & i & .12 \\
\hline 2.6 & ; & 2.63 & $:$ & 439 & : & 31.2 & i & 5.7 & $:$ & 4.9 & ; & .13 \\
\hline 2.8 & ! & 2.56 & i & 419 & $!$ & 32.6 & $:$ & 5.7 & $!$ & 4.9 & $:$ & .13 \\
\hline
\end{tabular}

All of these dipoles have essentially the same size and use similar quantities of $\mathrm{Nb}-\mathrm{Ti}$. The variation in current density comes from a different copper to superconductor ratio. 
First we commpare the two- and three-shell designs for the same field quality (at $j=670$ ). The two shell dipole is larger. The outside size is $4.6 \mathrm{~cm}$ instead of $4.0 \mathrm{~cm}$, and it uses $16 \%$ more superconductor, but the larger size is more than compensated by the simple construction. [It is difficult to find a practical example where the offset two-shell is not preferred!]

There is no great pressure to push the Cu:Nb-Ti ratio to the lowest possible value, however a higher current density does reduce the keystone to a reasonable level and eliminates any worry about random errors (and also persistent currents).

From the unequal shell designs in table IV we could make another table of possible dipoles. For comparison, a dipole with $\mathrm{j}=670$ would have $\mathrm{a}=2.90$ and would be $2 \mathrm{~mm}$ smaller and use $4 \%$ less superconductor than the simple two-shell; perhaps a useful modification for large scale production but the extra wire and cable sizes would be annoying at the modelling stage.

Dipole size, that is the outside size, is directly related to the required field quality. The primary use for higher current density is to make higher fields.

\section{Conclusions}

The offset shell designs extend the very practical two-shell doubler design to the higher fields and the higher quality necessary for larger, more complex proton rings.

The dominant consideration in choosing a dipole will be the systematic field quality.

New inventions are to be expected but there are boundaries to reasonable expectations for reduced cost and high quality. 
Table I. The Perfect Dipole

$B_{0}=\left[\mu_{0} j a / 2 \pi\right] b$

area $=\mathrm{qB}^{2}$

efficiency $f=b / q$

for comparison with later tables

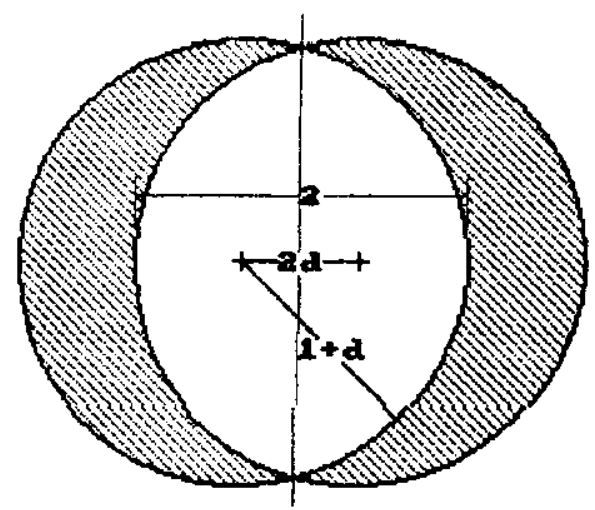

\begin{tabular}{|c|c|c|c|c|c|c|c|c|}
\hline b & & d & & $\mathbf{q}$ & & f & & $\mathbf{R}$ \\
\hline 1.2 & ! & .191 & : & 1.81 & ; & .662 & i & 1.38 \\
\hline 1.4 & $i$ & .223 & $:$ & 2.17 & ! & .646 & i & 1.44 \\
\hline 1.6 & $i$ & .255 & i & 2.54 & $:$ & .630 & i & 1.51 \\
\hline 1.8 & : & .286 & ! & 2.92 & ! & .616 & i & 1.57 \\
\hline 2.0 & $\begin{array}{l}: \\
:\end{array}$ & .318 & ! & 3.32 & $\begin{array}{l}! \\
\vdots\end{array}$ & .602 & $\begin{array}{l}1 \\
i\end{array}$ & 1.64 \\
\hline 2.2 & : & .350 & $!$ & 3.74 & : & .588 & $i$ & 1.70 \\
\hline 2.4 & : & . 382 & 1 & 4.17 & : & .576 & i & 1.76 \\
\hline 2.6 & $!$ & .414 & : & 4.61 & : & .564 & $i$ & 1.83 \\
\hline 2.8 & : & .446 & $:$ & 5.07 & $:$ & .552 & $i$ & 1.89 \\
\hline 3.0 & $:$ & .477 & $:$ & 5.54 & : & .541 & $i$ & 1.95 \\
\hline
\end{tabular}


Table II. Three Shell Dipoles, $2 d=3 w$ $B_{0}=\left[\mu_{0} j \mathbf{j} / 2 \pi\right] b$ area $=q a^{2}$ efficiency $f=b / q$ multipoles in a units

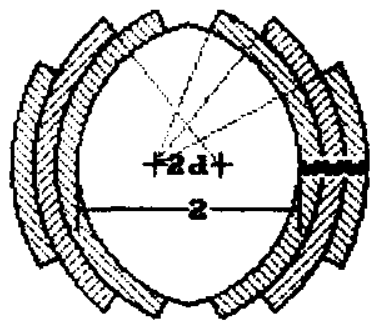

\begin{tabular}{|c|c|c|c|c|c|c|c|c|c|c|}
\hline$b$ & & $\mathbf{q}$ & & $f$ & & $\mathbf{R}$ & & $b_{18}$ & & $b_{2} 2$ \\
\hline 1.2 & : & 1.80 & i & .666 & i & 1.40 & $!$ & -.0066 & ! & +.0015 \\
\hline 1.4 & i & 2.16 & : & .649 & : & 1.46 & $i$ & -.0048 & 4 & +.0011 \\
\hline 1.6 & $\vdots$ & 2.53 & : & .633 & ! & 1.53 & $!$ & -.0035 & $:$ & +.0008 \\
\hline 1.8 & $i$ & 2.91 & ; & .618 & $\vdots$ & 1.59 & ; & -.0026 & $i$ & +.0006 \\
\hline 2.0 & $\begin{array}{l}i \\
i\end{array}$ & 3.31 & i & .604 & ! & 1.66 & : & -.0019 & i & +.0005 \\
\hline 2.2 & $:$ & 3.73 & i & .590 & : & 1.73 & $!$ & -.0015 & ; & +.0005 \\
\hline 2.4 & i & 4.16 & : & .577 & ? & 1.79 & $i$ & -.0011 & : & +.0005 \\
\hline 2.6 & $i$ & 4.60 & : & .565 & ! & 1.86 & $i$ & -.0009 & $\vdots$ & +.0005 \\
\hline 2.8 & $!$ & 5.06 & $i$ & .553 & : & 1.92 & : & -.0007 & : & +.0005 \\
\hline 3.0 & : & 5.53 & $!$ & .542 & 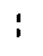 & 1.99 & $!$ & -.0005 & $i$ & +.0005 \\
\hline
\end{tabular}

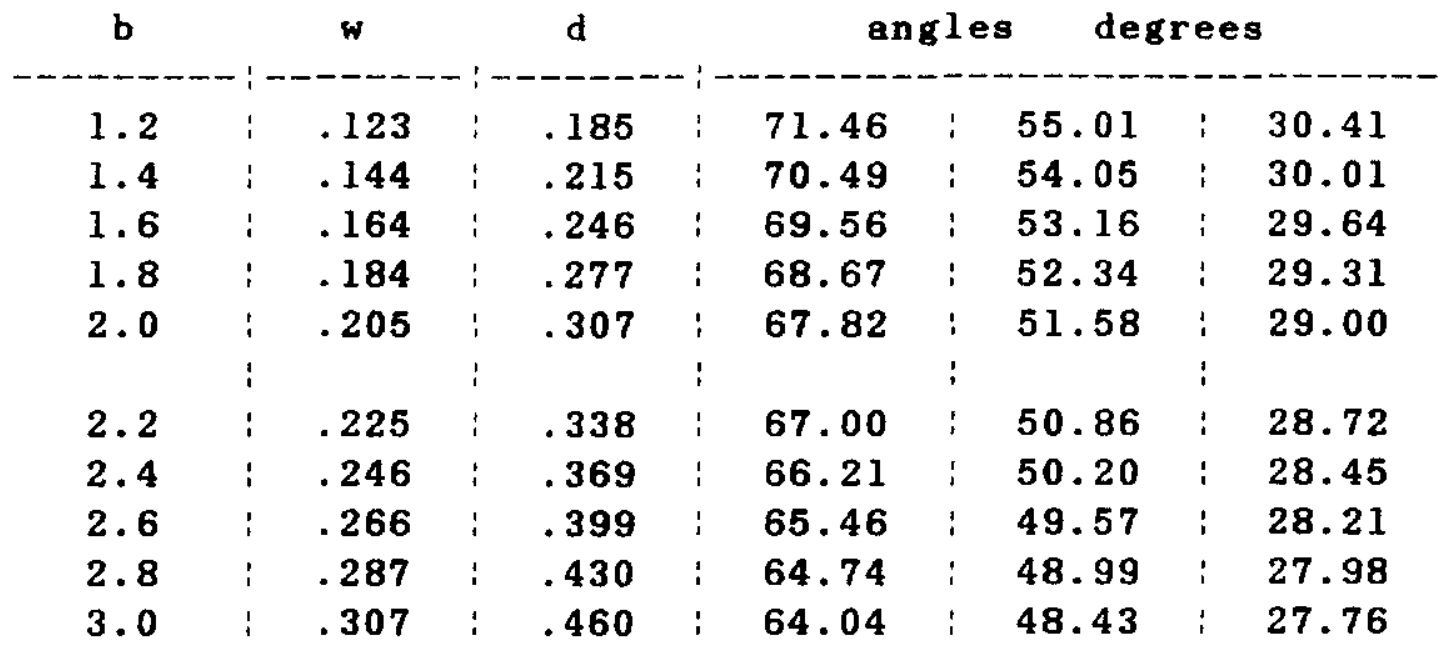


Table III. Two Shell Dipoles

$B_{0}=\left[\mu_{0} j a / 2 \pi\right] b$

area $=q \mathbf{a}^{2}$

efficiency $f=b / q$ multipoles in a units

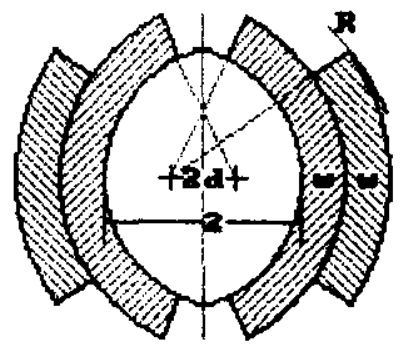

\begin{tabular}{|c|c|c|c|c|c|c|c|c|c|c|}
\hline b & & $\mathbf{q}$ & & $\mathbf{f}$ & & $\mathbf{R}$ & & $b_{1} a$ & & $b_{22}$ \\
\hline 1.2 & : & 1.73 & $!$ & .694 & $!$ & 1.38 & $!$ & -.0291 & : & +.0285 \\
\hline 1.4 & : & 2.08 & $i$ & .675 & $i$ & 1.45 & $!$ & -.0215 & : & +.0201 \\
\hline 1.6 & ; & 2.44 & $\vdots$ & .655 & $i$ & 1.52 & $:$ & -.0161 & $:$ & +.0145 \\
\hline 1.8 & $i$ & 2.83 & 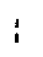 & .637 & i & 1.59 & i & -.0122 & $i$ & +.0106 \\
\hline 2.0 & $\begin{array}{l}i \\
\vdots\end{array}$ & 3.23 & $\begin{array}{l}\vdots \\
\vdots\end{array}$ & .620 & $\begin{array}{l}\vdots \\
\vdots\end{array}$ & 1.66 & $\vdots$ & -.0094 & $\begin{array}{l}\vdots \\
\vdots\end{array}$ & +.0078 \\
\hline 2.2 & ! & 3.64 & ! & .604 & : & 1.72 & : & -.0073 & $:$ & +.0059 \\
\hline 2.4 & : & 4.07 & $!$ & .589 & : & 1.79 & ! & -.0057 & $:$ & +.0044 \\
\hline 2.6 & : & 4.51 & $i$ & .575 & i & 1.86 & $i$ & -.0045 & $i$ & +.0034 \\
\hline 2.8 & : & 4.98 & $:$ & .562 & : & 1.93 & $i$ & -.0036 & $!$ & +.0027 \\
\hline 3.0 & $:$ & 5.46 & $:$ & .550 & : & 1.99 & $i$ & -.0029 & $\vdots$ & +.0021 \\
\hline
\end{tabular}

\begin{tabular}{|c|c|c|c|c|c|c|c|c|c|c|}
\hline \multicolumn{2}{|l|}{ b } & \multicolumn{2}{|l|}{$\omega$} & \multicolumn{2}{|l|}{ d } & \multicolumn{2}{|c|}{ angles } & \multicolumn{2}{|l|}{ deg. } & $k s t n$ \\
\hline 1.2 & : & .188 & $:$ & .017 & ! & 73.02 & $i$ & 38.82 & : & .085 \\
\hline 1.4 & $i$ & .219 & ; & .055 & $\mathrm{i}$ & 71.59 & : & 38.13 & : & .094 \\
\hline 1.6 & : & .249 & : & .092 & $\vdots$ & 70.25 & $i$ & 37.50 & $:$ & .102 \\
\hline 1.8 & $:$ & .280 & $:$ & .130 & $:$ & 68.99 & $:$ & 36.93 & $:$ & .110 \\
\hline 2.0 & $:$ & .310 & ; & .168 & : & 67.81 & $:$ & 36.41 & $i$ & .117 \\
\hline & $i$ & & $:$ & & $!$ & & 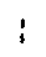 & & $!$ & \\
\hline 2.2 & $i$ & .340 & i & .207 & $:$ & 66.70 & $:$ & 35.93 & : & .123 \\
\hline 2.4 & $:$ & .370 & $i$ & .246 & ; & 65.65 & : & 35.48 & : & .129 \\
\hline 2.6 & ; & .400 & ! & .285 & : & 64.65 & ! & 35.07 & $\vdots$ & .135 \\
\hline 2.8 & $:$ & .429 & $:$ & .324 & i & 63.71 & : & 34.68 & $:$ & .139 \\
\hline 3.0 & : & .459 & $:$ & .363 & 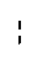 & 62.82 & $i$ & 34.33 & $:$ & .144 \\
\hline
\end{tabular}


Table IV. Two Unequal Shells *w2 $=.75 \mathrm{w}$ $B_{0}=\left[\mu_{0} j a / 2 \pi\right] b$

area $=\mathbf{q a}^{2}$

efficiency $f=b / q$ altipoles in $a$ units

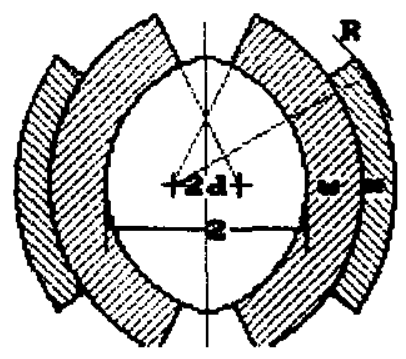

\begin{tabular}{|c|c|c|c|c|c|c|c|c|c|c|}
\hline b & & $q$ & & $f$ & & $\mathbf{R}$ & & $b_{1} a$ & & $b_{2} 2$ \\
\hline 1.2 & ! & 1.80 & $!$ & .668 & : & 1.40 & : & -.0167 & ; & +.0126 \\
\hline 1.4 & i & 2.16 & $i$ & .649 & : & 1.47 & $!$ & -.0123 & i & +.0089 \\
\hline 1.6 & ; & 2.54 & $i$ & .631 & ; & 1.54 & $:$ & -.0092 & i & +.0064 \\
\hline 1.8 & $i$ & 2.93 & : & .614 & $:$ & 1.60 & $i$ & -.0069 & ; & +.0047 \\
\hline 2.0 & $\begin{array}{l}i \\
\vdots\end{array}$ & 3.35 & : & .598 & i & 1.67 & $\begin{array}{l}i \\
i\end{array}$ & -.0053 & : & +.0034 \\
\hline 2.2 & 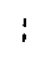 & 3.77 & $:$ & .583 & $:$ & 1.74 & ! & -.0041 & i & +.0025 \\
\hline 2.4 & $:$ & 4.22 & $:$ & .569 & : & 1.80 & $:$ & -.0033 & $i$ & +.0017 \\
\hline 2.6 & : & 4.68 & $i$ & .555 & $i$ & 1.87 & $!$ & -.0026 & ? & +.0010 \\
\hline 2.8 & $i$ & 5.16 & $:$ & .543 & $\vdots$ & 1.93 & $:$ & -.0022 & $i$ & +.0005 \\
\hline 3.0 & $i$ & 5.65 & $i$ & .531 & $i$ & 2.00 & $i$ & -.0019 & : & +.0001 \\
\hline
\end{tabular}

\begin{tabular}{|c|c|c|c|c|c|c|c|c|c|c|}
\hline \multicolumn{2}{|l|}{ b } & \multicolumn{2}{|l|}{$\mathbf{w}$} & \multicolumn{2}{|l|}{ d } & \multicolumn{2}{|c|}{ angles } & \multicolumn{2}{|l|}{ deg. } & $k=t n$ \\
\hline 1.2 & ; & .210 & $\vdots$ & .175 & : & 66.55 & $!$ & 34.14 & $:$ & .082 \\
\hline 1.4 & $!$ & .244 & : & .222 & i & 65.24 & i & 33.54 & ; & .091 \\
\hline 1.6 & ! & .278 & $\vdots$ & .268 & ; & 64.03 & $i$ & 33.00 & i & .099 \\
\hline 1.8 & i & .311 & i & .315 & i & 62.89 & i & 32.51 & i & .106 \\
\hline 2.0 & ; & .345 & $\begin{array}{l}\vdots \\
\vdots\end{array}$ & .362 & : & 61.82 & ; & 32.06 & : & .112 \\
\hline 2.2 & ! & .378 & $\vdots$ & .410 & $i$ & 60.81 & $i$ & 31.64 & $:$ & .118 \\
\hline 2.4 & i & .411 & $i$ & .457 & $i$ & 59.87 & $:$ & 31.26 & $i$ & .124 \\
\hline 2.6 & $:$ & .444 & $\vdots$ & .505 & i & 58.98 & $i$ & 30.91 & $i$ & .129 \\
\hline 2.8 & : & .478 & : & .552 & ; & 58.14 & 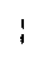 & 30.58 & : & .133 \\
\hline 3.0 & ; & .511 & i & .598 & $i$ & 57.36 & $!$ & 30.27 & : & .138 \\
\hline
\end{tabular}




\section{QUADRUPOLES}

Quadrupoles must also have high quality fields. This is obvious for the monster quads associated with low-beta straight sections, but it is also true for the modest normal-lattice quads. High multipoles are effective when particles are at their greatest displacement and that occurs in the quadrupoles and the ends of the dipoles next to the quads, thus the effect of quadrupole quality is disproportionately high. A full tracking program including all field errors is necessary to set precise limits.

We will follow a logical proceedure for quadrupole degign similar to that for dipoles, and use the same nomenclature except that

gradient $G=g\left[\mu_{0} j / 2 \pi\right]=g\left(a m p s / \mathrm{mm}^{2}\right) / 5$ tesla $/ \mathrm{m}$ and

$B_{y}=\left(\mu_{0} j a / 2 \pi\right) g\left[(x / a)+b_{5}(x / a)^{5}+b_{9}(x / a)^{9}+b_{13}(x / a)^{13}+b_{17}(x / a)^{17} \ldots\right.$

on the x-axis. (The multipole coefficients are measured in terms of the field $G a$ instead of $\left.B_{\circ}\right)$. The actual gradient does depend on a $(\sim \sqrt{a})$ because larger a means higher field at the coil and hence less current density.

The coil shape for a perfect quadrupole field from a uniform current density is shown in figure 7. It is created by an overlapping pair of ellipses which are shown with a minor axis of unity (to be scaled by a) and a major axis of $b$.

The relative parameters are

$$
\begin{gathered}
g=2 \pi(1-b) /(1+b) \\
q=4 b\left[\tan ^{-1}(b)-\tan ^{-1}(1 / b)\right] \\
f=g / q \\
R=b \\
r_{m}=\left(2 b^{2} /\left(1+b^{2}\right)\right)^{4 / 2}
\end{gathered}
$$

where $r=$ is the maximum inside radius (to the cross-over). The maximum field at the coil is

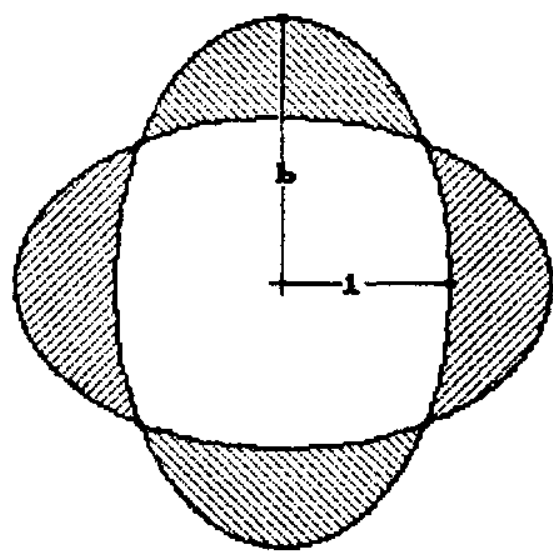

fig 7 The Perfect Quad proportional to rma. These formulae come from a straight-forward integration of the usual multipole expressions using radial limits which vary with $2 \vartheta$. This design is probably "well-known" and buried somewhere in the literature, otherwise it is an "original contribution". 
Table $\mathrm{V}$ is a convenient set of values for the perfect quad. We will find that any resonable quad has parameters which are not significantly different which makes this table actually very useful. For example suppose we want a strong quad with $G=250 \mathrm{t} / \mathrm{m}$ and $\mathrm{a}=3 \mathrm{~cm}$.

We can estimate that $r m=4 \mathrm{~cm}$ so the maximum field would be about 10 tesla! Using $g=3$ one would need $j=416 \mathrm{a} / \mathrm{mm}^{2}$, which just might be available at 10 tesla. On the other hand if the desired $G=140 \mathrm{t} / \mathrm{m}$, then Bear 5 and $\mathbf{g}=1, \mathrm{j}=700 \mathrm{a} / \mathrm{mm}^{2}$ should be easy (quads are never really easy). The first device has an outside coil diameter of $17 \mathrm{~cm}$ and a crosssection of $91 \mathrm{~cm}^{2}$, the second is $1 \mathrm{ess}$ than $8 \mathrm{~cm}$ and $16 \mathrm{~cm}^{2}$, much of the difference coming from the inevitable loss of efficiency with large $g$.

\section{Shell Designs}

Shells built from key-stoned cable, which worked so well for dipoles, produce poor results for quadrupoles. The simple two-shell angles can be adjusted to cancel the lower two multipoles ( $b_{5}, b_{9}$ ) only if the shell width w is less than .16, and the third multipole vanishes for $w=.069$. The introduction of offsets somewhat improves this range but the quadrupoles are still much to weak to be useful. The sharp edge needed near the cross-over is not well fitted by the wedges. Coils using "slipped stacks" fit much better.

Stacked coils use flat turns and have parallel outer edges. They are specified by the curve of the inner surface, which is shifted for adjacent stacks, and by the various stack half-heights (h1, $h_{2}$ etc.). The use of stacked coils greatly extends the range where lower multipoles can be set to zero but does not eliminate the problem. Larger values of $g$ (and $w$ ) will need more shells.

Table VI contains a set of stacked two-shell designs. The two heights, the width, and the offset were adjusted to obtain $g$ with the lower three multipoles zero. The inner surface does not correspond to the equivalent ellipse. This process works up to $g=2$. The higher multipoles are small and will have no effect on the beam at amplitudes less than $a / 2$.

Table V11 contains three shell designs which extend the range to $g=2.5$. They are intended for larger quads where the turn width would be excessive in the two-shell version. The inner surface has the theoretical offset and the higher multipoles are very small. 
Table VIII contains a few examples of four shell designs for even higher $g$ (and smaller $w$ ). The height of the fourth shell is chosen arbitrarily for a good fit to the ellipse. Although the higher multipoles are so small as to be meaningless, it is probable that we should limit amplitudes to $2 / 3$ of a because of construction errors. The parameters are now the same as the perfect coil.

\section{Practical Considerations}

The parallel surfaces of the collars will provide a pre-load to overcome the compressive magnetic forces, but there is no component firmly seating the coils against the collars before and during cool-down. For the thinner shells there may even be a tendency to bend the coil away from the collar.

The inner stacks almost fill the available space, particulaly for large $g$, which makes bending the ends of top turns very difficult. Unfortunately these turns are particularly important for field quality.

Construction problems for the normal quads can be eased by judiciously accepting poorer relative quality but preserving the performance by building somewhat larger quads. The cost of quads is almost wholly labor and is little affected by size. [Doubler quads were built larger but without offsets which should be effective.] This procedure cannot help the monster quads.

The relation between low beta and quadrupole gradient and size is well understood. Very low beta interaction regions so ease many design problems for storage rings that an optomistic anticipation of quadrupole strength is irresistable, particularly with no clearly stated limitations.

Based on the perfect quad parameters and with a realistic assessment of overall current density (particulaly at high fields) one can construct a table of maximum gradient versus size for practical but difficult quads. The real design will not have substantially different performance, in particular systematic field quality will be good and probably $2 / 3$ of the width is useful. This transfers the problem of the monster quads back to the lattice designers and they can back off from very low betas if necessary.

\section{Conclusion.}

The study of perfect quads and the approximation by stacked coils leaves a number of difficult design problems, but it does clearly show that high gradients and high quality are achievable within well defined parameters. 
Table $V$ The Perfect Quadrupole

$$
\begin{aligned}
& \text { Grad. }=g\left[\mu_{0} j / 2 \pi\right] \\
& \text { coil area }=q a^{2} \\
& \text { efficiency } f=g / q \\
& \text { outside size }=R a \\
& \text { inside size }=a
\end{aligned}
$$

\begin{tabular}{|c|c|c|c|c|c|c|c|c|c|c|}
\hline 8 & & $\mathbf{q}$ & & $\mathbf{f}$ & & $* d$ & & $\star * \mathbf{r}=$ & & $\mathbf{R}, \mathbf{b}$ \\
\hline .6 & $!$ & .92 & : & .650 & : & .41 & ; & 1.09 & : & 1.211 \\
\hline .8 & ! & 1.31 & : & .611 & : & .60 & $\mathfrak{a}$ & 1.12 & $\vdots$ & 1.292 \\
\hline 1.0 & $\begin{array}{l}i \\
i\end{array}$ & 1.74 & $\begin{array}{l}i \\
i\end{array}$ & .575 & : & .82 & ! & 1.14 & $\begin{array}{l}\text { i } \\
\text { : }\end{array}$ & 1.379 \\
\hline 1.2 & $:$ & 2.22 & $:$ & .540 & : & 1.07 & $:$ & 1.17 & ! & 1.472 \\
\hline 1.4 & ; & 2.76 & $:$ & .507 & $!$ & 1.36 & i & 1.19 & $:$ & 1.573 \\
\hline 1.6 & $:$ & 3.36 & $\vdots$ & .477 & : & 1.71 & : & 1.22 & ; & 1.683 \\
\hline 1.8 & : & 4.02 & $:$ & .447 & : & 2.11 & : & 1.24 & $i$ & 1.803 \\
\hline 2.0 & $\begin{array}{l}i \\
i\end{array}$ & 4.77 & $\begin{array}{l}\text {; } \\
\text { : }\end{array}$ & .419 & : & 2.59 & : & 1.26 & $\begin{array}{l}i \\
i\end{array}$ & 1.934 \\
\hline 2.2 & $!$ & 5.60 & : & .393 & ! & 3.15 & ; & 1.27 & $:$ & 2.078 \\
\hline 2.4 & $i$ & 6.53 & $i$ & .368 & ; & 3.83 & : & 1.29 & i & 2.236 \\
\hline 2.6 & $:$ & 7.57 & ! & .343 & i & 4.63 & ${ }^{\prime}$ & 1.31 & $:$ & 2.412 \\
\hline 2.8 & $i$ & 8.75 & : & .320 & : & 5.61 & $i$ & 1.32 & : & 2.608 \\
\hline 3.0 & $:$ & 10.08 & : & .298 & : & 6.80 & i & 1.33 & : & 2.828 \\
\hline
\end{tabular}

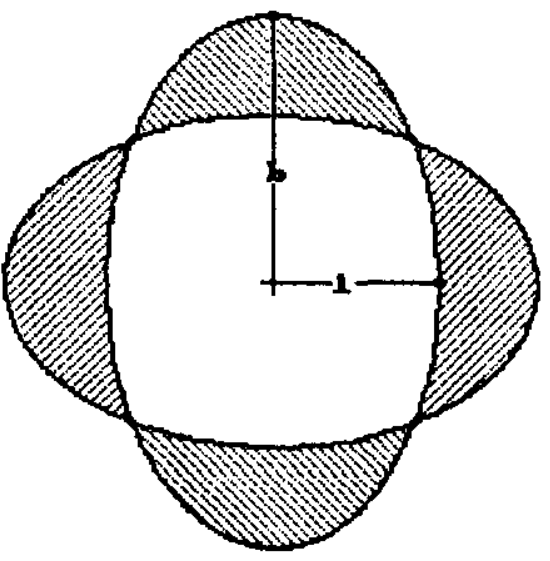

* the inner surface is well approximated by a circular arc with offset $d$. ** the radius to the cross-over is ra. Gran is the highest field. 
Table VI Offset Two-Shell Quadrupoles

$$
\begin{aligned}
& \text { Grad. }=g[\mu \circ j / 2 \pi] \\
& \text { coil area }=q a^{2} \\
& \text { efficiency } f=g / q \\
& \text { outside size }=R a \\
& \text { inside size }=a
\end{aligned}
$$

\begin{tabular}{|c|c|c|c|c|c|c|c|c|}
\hline $\mathbf{g}$ & & $\boldsymbol{w}$ & & d & & $h_{1}$ & & $h_{2}$ \\
\hline .6409 & i & .114 & i & 0 & i & .630 & i & .398 \\
\hline .8 & $i$ & .145 & : & .189 & ; & .662 & $i$ & .419 \\
\hline 1.0 & : & .184 & : & .488 & : & .702 & ; & .447 \\
\hline 1.2 & $\vdots$ & .226 & : & .882 & : & .740 & : & .476 \\
\hline 1.4 & $:$ & .269 & : & 1.41 & ; & .778 & : & .507 \\
\hline 1.6 & : & .314 & $i$ & 2.14 & : & .814 & $!$ & .540 \\
\hline 1.8 & : & .361 & ; & 3.19 & $i$ & .848 & $i$ & .574 \\
\hline 2.0 & $i$ & .410 & : & 4.78 & $:$ & .880 & $:$ & .611 \\
\hline
\end{tabular}

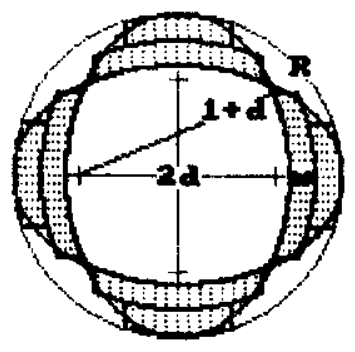

multipoles use a field Ga and units of a

$$
g=1.2
$$

\begin{tabular}{c:ccccc}
8 & $q$ & $f$ & $R$ & $b_{17}$ \\
\hdashline 6409 & .94 & .682 & 1.21 & -.0213 \\
.8 & 1.25 & .639 & 1.28 & -.0102 \\
1.0 & 1.69 & .590 & 1.38 & -.0042 \\
& & & & & \\
1.2 & 2.20 & .547 & 1.47 & -.00175 \\
1.4 & 2.76 & .507 & 1.57 & -.00075 \\
1.6 & 3.40 & .471 & 1.67 & -.00033 \\
1.8 & 4.10 & .439 & 1.78 & -.00015 \\
2.0 & 4.89 & .409 & 1.89 & -.00007
\end{tabular}

$h_{1}$ and $h_{2}$ are half-heights of the stacks of flat turns 
Table VII Offset Three-Shell Quadrupoles

$$
\begin{aligned}
& \text { Grad. }=g\left[\mu_{0} j / 2 \pi\right] \\
& \text { coil area }=q a^{2} \\
& \text { efficiency } f=g / q \\
& \text { outside size }=R a \\
& \text { inside size }=a
\end{aligned}
$$

\begin{tabular}{|c|c|c|c|c|c|c|c|c|c|}
\hline 8 & & $q$ & & f & & $\mathbf{R}$ & & $b_{1} 7$ & 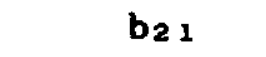 \\
\hline 1.0 & $i$ & 1.74 & i & .576 & ! & 1.38 & i & -.000582 & $:+.000061$ \\
\hline 1.2 & : & 2.22 & i & .541 & : & 1.47 & $i$ & -.000201 & $:+.000058$ \\
\hline 1.4 & $!$ & 2.76 & : & .508 & ! & 1.57 & $i$ & -.000089 & $:+.000065$ \\
\hline 1.6 & $:$ & 3.36 & ! & .477 & $!$ & 1.68 & $i$ & -.000060 & $:+.000060$ \\
\hline 1.8 & $i$ & 4.02 & $\vdots$ & .447 & : & 1.79 & $\vdots$ & -.000052 & $:+.000049$ \\
\hline 2.0 & $!$ & 4.77 & : & .419 & $i$ & 1.92 & $\vdots$ & -.000045 & $:+.000036$ \\
\hline 2.2 & ; & 5.60 & $:$ & .393 & $!$ & 2.04 & $i$ & -.000038 & $:+.000025$ \\
\hline 2.4 & $!$ & 6.53 & $\vdots$ & .367 & ! & 2.18 & ! & -.000030 & $i+.000017$ \\
\hline 2.6 & $:$ & 7.58 & : & .343 & $\vdots$ & 2.32 & ; & -.000022 & $i+.000011$ \\
\hline
\end{tabular}

\begin{tabular}{|c|c|c|c|c|c|c|c|c|c|c|}
\hline $\mathbf{8}$ & & $\boldsymbol{w}$ & & d & & $\mathbf{h}_{1}$ & & $h_{2}$ & & $\mathbf{h}_{3}$ \\
\hline 1.0 & : & .122 & $:$ & .815 & : & .760 & : & .628 & : & 390 \\
\hline 1.2 & : & .152 & $i$ & 1.07 & $i$ & .779 & ! & .641 & $i$ & .404 \\
\hline 1.4 & $i$ & .184 & $!$ & 1.36 & $i$ & .798 & $:$ & .655 & $:$ & .418 \\
\hline 1.6 & : & .219 & $:$ & 1.70 & : & .815 & $:$ & .668 & i & .433 \\
\hline 1.8 & : & .256 & i & 2.11 & i & .831 & : & .681 & : & .451 \\
\hline 2.0 & $:$ & .296 & ! & 2.59 & : & .847 & $!$ & .694 & $i$ & .475 \\
\hline 2.2 & : & .337 & : & 3.15 & $i$ & .862 & : & .705 & : & .511 \\
\hline 2.4 & i & .379 & ! & 3.83 & : & .877 & : & .716 & $:$ & .562 \\
\hline 2.6 & $i$ & .421 & i & 4.63 & $!$ & .891 & : & .724 & : & .635 \\
\hline
\end{tabular}

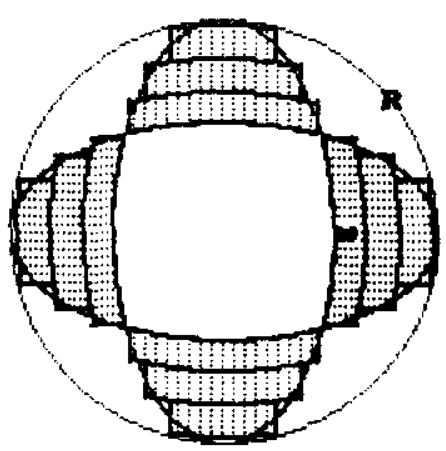

multipoles ise a field Ga and units of a

$$
g=2
$$


Table VIII Offset Four-Shell Quadrupoles

$$
\begin{aligned}
& \text { Grad. }=g\left[\mu_{0} j / 2 \pi\right] \\
& \text { coil area }=q a^{2} \\
& \text { efficiency } f=g / q \\
& \text { outside size }=R a \\
& \text { inside size }=a
\end{aligned}
$$

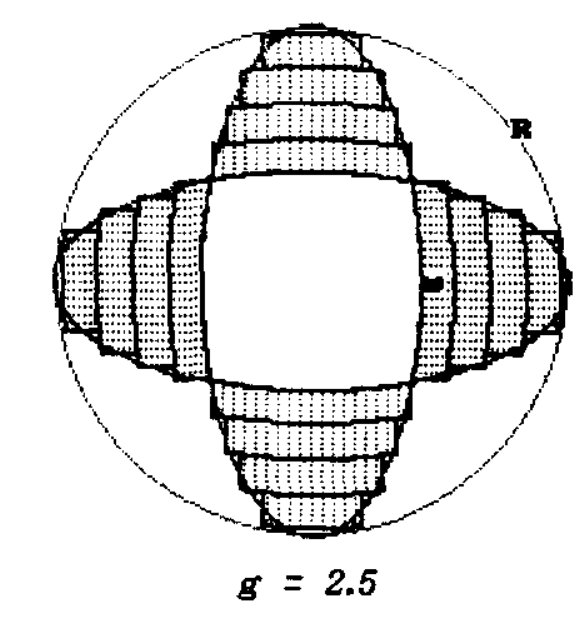

multipoles use a field Ga and units of $a$

\begin{tabular}{c:ccccc}
$g$ & $q$ & $f$ & $R$ & $b_{17}$ & $b_{21}$ \\
\hdashline 2.0 & 4.77 & .419 & 1.95 & -.000001 & +.000009 \\
2.5 & 7.04 & .355 & 2.29 & -.000009 & +.000006 \\
3.0 & 10.06 & .298 & 2.71 & -.000006 & +.000003
\end{tabular}

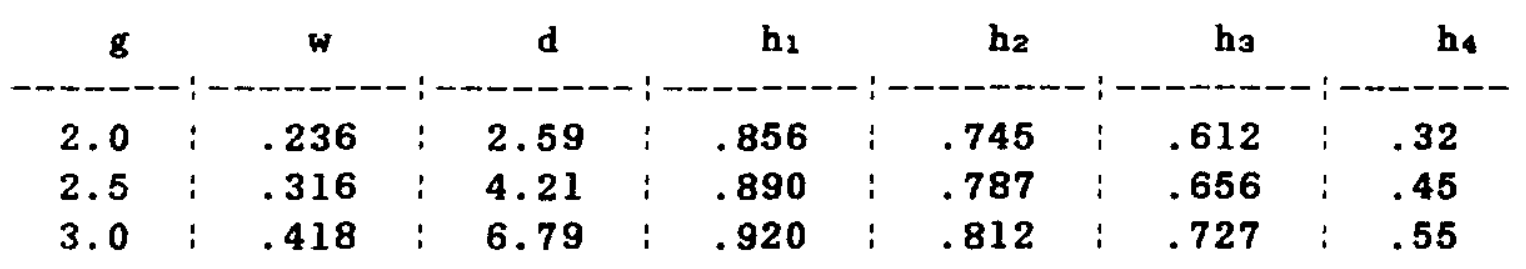

